\title{
Laryngeal Cartilage Invasion
}

$\mathrm{n}$ their article comparing MR imaging and dual-energy CT for the evaluation of cartilage invasion by laryngeal carcinoma, ${ }^{1}$ the authors correctly stated in their introduction that the diagnosis of T4a disease - that is, tumor invading not just into but beyond the laryngeal cartilage and into the extralaryngeal soft tissuesconstitutes a potential indication for laryngectomy. However, what follows appears to be an exercise in establishing whether there is merely tumor infiltration into the cartilage, a finding that may only establish T3 disease and that would not constitute grounds for surgery. Indeed, when the treatment goal is larynx conservation, the determination of tumor just within the cartilage is not critical, and whether dual-energy CT is better than MR imaging may be moot. In fact, the only imaging example pre- sented in this article, Fig 1, shows a case in which there is no suggestion of extralaryngeal spread on either MR imaging or dualenergy CT. Much has been written on imaging of cartilage extension in laryngeal cancer, little of which, in my opinion, has bearing on real-world treatment, given the goal of organ preservation, in which laryngectomy is reserved for only advanced disease.

\section{REFERENCE}

1. Kuno H, Sakamaki K, Fujii S, et al. Comparison of MR imaging and dual-energy CT for the evaluation of cartilage invasion by laryngeal and hypopharyngeal squamous cell carcinoma. AJNR Am J Neuroradiol 2018;39:524-31 CrossRef Medline 